\title{
AMENABILITY AND THE SPECTRUM OF THE LAPLACIAN
}

\author{
BY ROBERT BROOKS ${ }^{1}$
}

In this note, we announce some results $[1,2]$ relating the notion of amenability of a group with spectral geometry. These results take their inspiration from the theorem of Milnor-Švarč [5], which relates the growth of the fundamental group in the sense of group theory with the growth of the universal cover in the sense of Riemannian geometry. Our first result interprets the amenability of the fundamental group of a compact manifold $M$ in terms of a spectral condition on the universal cover $\widetilde{M}$.

THEOREM 1. $\pi_{1}(M)$ is an amenable group if and only if 0 is in the spectrum of the Laplacian on $L^{2}(\widetilde{M})$.

We observe that although the spectrum of the Laplacian on $\widetilde{M}$ depends on a choice of metric on $M$, all such choices are quasi-isometrically equivalent; hence from the variational expression

$$
\lambda_{0}(\widetilde{M})=\inf _{f} \frac{\int_{\widetilde{M}}\|\operatorname{grad} f\|^{2}}{\int_{\widetilde{M}} f^{2}}
$$

where $f$ runs over smooth functions with compact support, we see that the condition $\lambda_{0}(\widetilde{M})=0$ is independent of the choice of metric on $M$.

The proof of Theorem 1 revolves around the following characterization of amenable groups, due to Følner [4]. For those unfamiliar with the notion of amenability, one may take this as a definition:

THEOREM (FøLNER). Let $G$ be a finitely generated group, with generators $g_{1}, \ldots, g_{n}$. Then $G$ is amenable if and only if, for every $\epsilon$, there is a finite subset $E$ of $G$ such that

$$
\#\left(E \cap g_{i} \cdot E\right) \geqslant(1-\epsilon) \#(E) \text { for all } i .
$$

The proof of Theorem 1 proceeds by interpreting the F $\phi$ lner condition as an "isoperimetric condition" on the group $G$. In particular, we choose a fundamental domain $F$ for the action of $\pi_{1}(M)$ on $\widetilde{M}$. Then $\pi_{1}(M)$ is amenable if

Received by the editors April 8, 1981.

1980 Mathematics Subject Classification. Primary $58 \mathrm{G} 25$.

${ }^{1}$ Partially supported by NSF grant MCS7802679. 
and only if there is a sequence $E_{i}$ of unions of finitely many translates of $F$ by $\pi_{1}(M)$, such that the isoperimetric ratio

$$
\operatorname{area}\left(\partial E_{i}\right) / \operatorname{vol}\left(E_{i}\right)
$$

tends to 0 .

If $\pi_{1}(M)$ is amenable, then one uses the $E_{i}$ to construct test functions $f_{i}$ such that $\operatorname{grad} f_{i}$ is supported near $\partial E_{i}$, while $f_{i} \equiv 1$ inside $E_{i}$. Since, by F $\phi$ lner's condition, the area of $\partial E_{i}$ is small compared to $\operatorname{vol}\left(E_{i}\right)$, this can be done so that the ratio $\int_{\widetilde{M}}\left\|\operatorname{grad} f_{i}\right\|^{2} / \int_{\widetilde{M}} f_{i}^{2} \rightarrow 0$, showing $\lambda_{0}(\widetilde{M})=0$.

Conversely, if $\lambda_{0}(\widetilde{M})=0$, an estimate of Cheeger [3] says that the isoperimetric ratio $h=\inf _{N}(\operatorname{area}(\partial N) / \operatorname{vol}(N))$ tends to zero, where the $N$ are open submanifolds of $\widetilde{M}$ with compact closure. The idea of the remainder of the proof is to show that $\operatorname{area}(\partial N) / \operatorname{vol}(N)$ is estimated, up to a multiplicative constant, by replacing $N$ by a finite union of fundamental domains, establishing the amenability of $\pi_{1}(M)$. See [1] for details.

Theorem 1 can be generalized to the study of the spectral geometry of leaves in a foliation of a compact manifold. To state this generalization, let $F$ be a codimension $q$ foliation of a compact manifold $M$, and let $\left\{U_{i}\right\}$ be a finite covering of $M$ by flow boxes: $U_{i}$ is diffeomorphic to $D^{q} \times D^{n-q}$, in such a way that the leaves of the foliation are of the form $x \times D^{n-q}, x \in D^{q}$. Let $T_{i}=$ $D^{q} \times 0 \subset U_{i}$, and let $T=U T_{i} ; T$ is a transversal to the foliation $F$.

There is then a natural pseudogroup $\Gamma$ of partial diffeomorphisms of $T$, generated by the finite set $\left\{\Gamma_{i j}: U_{i} \cap U_{j} \neq \varnothing\right\}$ where $\Gamma_{i j}$ maps $\left\{x \in T_{i}\right.$ : $x \times D^{n-q}$ intersects $\left.U_{j}\right\}$ to $T_{j}$ by transporting along the leaves.

Theorem 2. Let $x \in T$, and denote by $L_{x}$ the leaf through $x$. Then $\lambda_{0}\left(L_{x}\right)=0$ if and only if, for all $\epsilon$, there is a finite set $E$ contained in the orbit of $x$ under $\Gamma$, such that

$$
\#\left\{y \in E: \Gamma_{i j}(y) \notin E \text { for some } i, j\right\}<\epsilon \cdot \#(E) \text {. }
$$

We call $x \in T$ a F $\phi$ lner point, and $L_{x}$ a F $\phi$ lner leaf, if $x$ satisfies the condition given in Theorem 2. As in Theorem 1, the above condition is equivalent to an isoperimetric condition on $L_{x}$. Clearly the set of F $\phi$ lner points is a Borel subset of $T$. Thus

Corollary 1. Suppose $F$ is an ergodic foliation of $M$ (with respect to the Riemannian measure on $M)$. Then either almost all leaves $L_{x}$ satisfy $\lambda_{0}\left(L_{x}\right)$ $=0$ or almost all leaves satisfy $\lambda_{0}\left(L_{x}\right)>0$. 
It follows from the proof of Theorem 2 that

COROLlARY 2. Let $L$ be a complete, noncompact Riemannian manifold such that the isoperimetric constant $h(L)=0$, but $\lambda_{0}(L)>0$. Then $L$ is not the leaf of a foliation of a compact manifold.

The proof of Theorem 2 follows closely the proof of Theorem 1, and is given in [2].

We remark also that many theorems about foliations with polynomial growth (e.g. $[6,7])$ generalize easily to foliations all of whose leaves are F $\phi$ lner.

ACKNowledgements. It is a great pleasure to thank J. Cheeger, B. Lawson, S. Wolpert, and R. Zimmer for helpful conversations and correspondence during the development of this work.

\title{
REFERENCES
}

1. R. Brooks, The fundamental group and the spectrum of the Laplacian, Comment. Math. Helv. (to appear).

2. The spectral geometry of foliations (to appear).

3. J. Cheeger, A lower bound for the smallest eigenvalue of the Laplacian, Problems in Analysis (Gunning, (ed.)), Princeton Univ. Press, Princeton, N. J., pp. 195-199. 243-254.

4. E. Folner, On groups with full Banach mean value, Math. Scand. 3 (1955),

5. J. Milnor, A note on curvature and fundamental group, J. Differential Geom. 2 (1968), 1-7.

6. J. Plante, A generalization of the Poincaré-Bendixson Theorem for foliations of codimension 1, Topology 12 (1973), 177-181.

7. C. Series, Foliations of polynomial growth are hyperfinite, Israel J. Math. 34 (1979), 245-258.

\author{
INSTITUTE FOR PHYSICAL SCIENCE AND TECHNOLOGY, UNIVERSITY OF \\ MARYLAND, COLLEGE PARK, MARYLAND 20742 \\ DEPARTMENT OF MATHEMATICS, UNIVERSITY OF MARYLAND, COLLEGE \\ PARK, MARYLAND 20742
}


